\title{
Evolutionary Multi-Objective Generation of Recurrent Neural Network Ensembles for Time Series Prediction
}

\author{
Christopher Smitha ${ }^{\mathrm{a}}$ Yaochu Jin ${ }^{\mathrm{a}, *}$ \\ ${ }^{a}$ Department of Computing, University of Surrey, Guildford, GU2 7XH, United Kingdom
}

\begin{abstract}
Ensembles have been shown to provide better generalization performance than single models. However, the creation, selection and combination of individual predictors is critical to the success of an ensemble, as each individual model needs to be both accurate and diverse. In this paper we present a hybrid multi-objective evolutionary algorithm that trains and optimizes the structure of recurrent neural networks for time series prediction. We then present methods of selecting individual prediction models from the Pareto set of solutions. The first method selects all individuals below a threshold in the Pareto front and the second is based on the training error. Individuals near the knee point of the Pareto front are also selected and the final method selects individuals based on the diversity of the individual predictors. Results on two time series data sets, Mackey-Glass and Sunspot, show that the training algorithm is competitive with other algorithms and that the final two selection methods are better than selecting all individuals below a given threshold or based on the training error.
\end{abstract}

Keywords:

Hybrid multi-objective evolutionary algorithms, Time series prediction, Ensembles, Selection, Recurrent neural networks

\section{Introduction}

Time series data can be regarded as any information that varies with time and time series forecasting can be described as projecting this time series data into the future

${ }^{*}$ Corresponding author 
[1]. Understanding the behavior of a dynamic system and using this information to 5 predict its future behavior can be very useful and it has been applied to the prediction of electricity demand [2], solar data [3], finance forecasting [4, 5] and hydrological forecasting [6].

To confidently predict time series data the dynamics of the system needs to be accurately modeled. Recurrent neural networks (RNNs) are considered suitable for time series prediction as they have both feed-back as well as feed-forward connections. A form of memory is incorporated into the networks, with the states of the neurons from previous iteration steps being stored and used to influence the prediction of data at future iterations. An ensemble of predictors can be used to produce a confident prediction and involves combining many different models to give the final prediction.

15 An ensemble can include information that is not contained in a single model [7] and each member can produce different errors. Ensembles have been shown to provide better generalization performance than single models and the result is a more confident final prediction [8]. Ensembles of RNNs should therefore be well suited for time series prediction.

Evolutionary algorithms (EA) use a population of solutions to solve a problem, which makes them ideal for creating potential individual predictors in an ensemble. EAs have been used to train neural networks [9, 10, 11, 12], however, some members in the final population may not be suitable, which means selecting the right models to ensemble is very important.

There has not been much discussion in the literature on how to select ensemble members from the Pareto set of solutions. For example in [13], two slightly different methods for generating ensemble members were reported. The first variant splits training data into two subsets, and the training error on the two subsets are used as two objectives. In the second variant, random noise is added to the training dataset to form the second objective. However, all Pareto-optimal solutions are used to construct ensembles and no selection strategy has been discussed. Therefore, this paper investigates different selection methods for time series prediction tasks. A hybrid multi-objective evolutionary algorithm (H-MOEA) is used to train RNNs and determine their optimal structure and four selection methods are investigated to select individual models 
from a filtered population of solutions. Firstly, all filtered members are selected. Then members with a low training accuracy and members located near the knee point of the Pareto front are considered. Finally, members with a large degree of diversity in the filtered population are selected. The normalized performance gain (NPG) [14] and the ambiguity term of the error-ambiguity decomposition [15] are used to determine the

40 knee point and the diversity terms, respectively.

Two time series data sets are used to test the training algorithm and selection methods. The Mackey-Glass is a simulated chaotic time series, while the Sunspot is a realworld time series. The prediction performance is compared with a number of other methods in the literature that have used similar parameter settings.

The rest of the paper is organized as follows. Background of recurrent neural networks and their training methods are presented in Section 2. Information on the specific training and selection algorithms are presented in Section 3 . Section 4 presents the experiments, results and discussions. Section 5 concludes the paper and provides details of future work.

\section{RNNs for Time Series Prediction}

\subsection{Time Series Data and Dynamic Reconstruction}

Univariate time series data is any dataset with only one variable. Dynamic reconstruction is concerned with establishing a model that "captures the underlying dynamics" of a system that uses univariate data and can be used to determine future values [16]. The reconstruction vector, $y_{R}(n)=[y(n), y(n-\tau), . ., y(n-(D-1) \tau)]^{T}$, is defined in terms of the time series observable output $y(n)$ and its delayed versions [16]. $\tau$ is the normalized embedding delay. $D$ corresponds to the minimum number of data points needed for dynamic reconstruction and is known as the embedding dimension.

Taken's Theorem states that $D \geq 2 d+1$, where $d$ is the dimension of the state space of the system [16]. The value of $D$ may not be known and although increasing $D$ can improve prediction, it could also introduce noise or imperfections into the system. It is therefore desirable to keep $D$ to a minimum. Various values for the embedding delay and dimension have been used for time series prediction; $D=3 / \tau=2[17], D=4 / \tau=6$ 
[18] and $D=3 / \tau=7$ [19] and values have also been determined using an evolutionary algorithm [20]. However, specific parameter values can be problem dependent.

Feed-forward neural networks [6, 21], recurrent radial basis functions [22] and fuzzy models [23] have all been used for time series prediction and have used various embedding delay and dimension values.

\subsection{Recurrent Neural Networks}

Neural networks are nonlinear models used to approximate solutions to complex problems and can be used to model any nonlinear function. They acquire knowledge of the system or environment they are embedded in through observations and use them to train the network [16]. Recurrent neural networks (RNNs) are dynamical systems that are specifically designed for temporal problems, as they have both feed-back as well as feed-forward connections. The overall structure of a RNN consists of synaptic connections between the input, hidden and output layers of neurons. Knowledge is represented in a network by the values of these synaptic connections. The states of the neurons are dependent on these free parameters, the inputs to the neurons and the states of the neurons at previous time steps [24]. A RNN can have copies of any neuron in the network from the previous time-step and they can be used to influence the prediction of data at future iterations. The objective of learning is to train the network by adjusting the connection weight values, over several training epochs, to reduce the output error of the network. Training moves the error towards a minimum point on the error surface, which has the free parameters of the network as its coordinates [16].

Gradient descent (GD) [19, 25], single-objective evolutionary algorithms (SOEA) [26, 27] and multi-objective evolutionary algorithms (MOEA) [20, 28, 29] have been used to train RNNs. Hybrid approaches to neural network training that combine global and local search techniques have also been used [30, 31, 32]. Of these hybrid methods, some have used Baldwinian [30] and others Lamarkian learning [33]. The global search is used to find suitable starting weight values and the local search to fine tune them to their optimal value. As well as training the networks weight values, MOEAs can also optimize the structure (number of connections) of the network [11, 34]. 


\subsection{Ensemble Member Generation and Selection}

When constructing an ensemble it is important that each individual model is both

95 [36] and this is summarized by the error-ambiguity decomposition presented by Krogh and Vedelsby [15]. Equation (11) summarizes this relationship, showing that the generalization error of an ensemble $(E)$ is based on the weighted average of the individual generalization errors $(\bar{E})$ and the weighted average of ambiguities $(\bar{A})$.

$$
E=\bar{E}-\bar{A}
$$

By reducing each individual's generalization error and increasing their ambiguity, the overall generalization error of the ensemble will reduce. However, by increasing the ambiguity of an individual predictor there is an increase in the individual's error.

Diverse ensemble members can be either implicitly or explicitly created. Different data samples, network parameters and initialization methods, as well as using different learning algorithms have all been used to implicitly create diverse ensemble members [35, 36]. To explicitly create diverse neural network ensembles, the ADDEMUP [12], DIVACE [10] and regularization [11] algorithms have all been used.

The use of an MOEA to create diverse ensemble members is very attractive, as the fitness functions can be specifically chosen to optimize conflicting objectives, with the resultant Pareto-optimal solutions providing a trade-off between these objectives and a set of optimal solutions [37].

There may be one model in the Pareto set that is able to perform better than an ensemble, however, there is no clear way of selecting this individual model [36]. There may also be unsuitable/infeasible solutions in the final Pareto set, so a subset may provide better performance and it has been suggested that to ensemble many of the individual members can be better than ensembling them all [38]. Therefore, an MOEA can be used as an indicator of which solutions to use in the ensemble and MOEAs have been used to successfully design neural networks for a variety of problems [7, 14].

Ranking the individual models, based on some criteria or through the use of an optimization process, have all been suggested as possible methods of selection [35]. 
The method used to combine the selected ensemble members is also very important and as stated in [35], the variance as well as the bias of learning algorithms may be reduced through an optimal combination.

The mean of a number of predictors [6, 39] or the weighted mean [4, 6] of the final output has been used to combine the individual predictors. The weighted median [40] and weighted sum [41] have also been used, among many others.

Section 3 provides information on the specific H-MOEA and selection algorithms used in this work.

\section{H-MOEA and Ensemble Member Selection}

\subsection{H-MOEA for Generation of RNNs}

The H-MOEA for generating RNNs consists of a multi-objective evolutionary algorithm for global search of both the weights and connectivity and a gradient-based local search for fine-tuning of the weights. Evolutionary algorithms (EAs) can be considered as multi-point search strategies that are able to sample a large search space and escape local optima to find global optimum solutions [42]. EAs are stochastic search and optimization procedures that are based on the principles of natural genetics and natural selection [43]. A population of individual candidates is used, instead of one candidate solution and new solutions are created by selection, crossover and mutation operators, during a set number of generations [42]. The specific design variables that make up a solution are coded into a chromosome, which is decoded to give a fitness/quality score of how well the individual satisfies the objective function(s). Selection, based on this score, is used to determine which individuals will be used as parents to create new offspring or to determine those that will be selected for the next generation.

In this work, each RNN in the population is encoded using two chromosomes. The first of a Boolean type to represent the structure of the networks and the second of real values to represent the weights of the networks. Fig. 1 1illustrates how the two chromosomes are linked and that specific alleles represent specific connections. This means that when a Boolean connection is present, the corresponding weight value is used by 
the network. The direct method of representing the network structure, as described in [34], is used, with every possible connection represented in the chromosomes.

\begin{tabular}{|l|c|c|c|c|c|c|c|c|c|}
$\begin{array}{l}\text { Chromosome 1 } \\
\text { Chromosome 2 }\end{array}$ & 1 & 1 & 0 & 1 & 1 & 0 & 1 & $\cdots$ & 1 \\
\cline { 2 - 9 } & -0.05 & 2.65 & 1.53 & 5.97 & 0.49 & 0.04 & -0.29 & $\ldots$ & -4.47 \\
\hline
\end{tabular}

Figure 1: Chromosomes for each recurrent neural network

The chromosomes are decoded to represent an individual network by placing the values of specific alleles into particular locations in the network structure. The topology of the networks is restricted to three input neurons, five hidden neurons and one output neuron. The states of the neurons from the previous time step are recalled and recurrent connections are allowed across all layers of neurons.

Fig. 2 is an example of the matrix setup used in this work, with locations below the main diagonal of the matrix representing forward connections and locations above the diagonal representing recurrent connections. Locations on the diagonal represent selfrecurrence. Therefore, when a connection, $C_{i j}$, equals 1 , a connection is made from neuron $j$ to neuron $i$. This means neuron $j$ is the connection start point and neuron $i$ the connection end point, i.e. neuron $i$ is receiving activation from neuron $j$. The connection highlighted in Fig. 2 shows that hidden neuron $1\left(H_{1}\right)$ receives activation from input neuron $3\left(I_{3}\right)$. Fig. 3illustrates the network presented in Fig. 2, with the state of the neurons from the previous time step represented by the grey dotted circles. The solid arrows represent forward connections and the dotted arrows represent recurrent connections, which originate from the neurons at $(t-1)$.

The two conflicting objectives used by the EA are the mean squared error (MSE) on a training data set and the number of connections (NC) in the network. Both are minimized and this is because large complexity is the main reason behind over-fitting [11]. Batch learning is used, with all data presented to the network before the training MSE is calculated, minus a warm-up-length. A warm-up-length of data is taken into consideration during batch learning and is used to initialize the internal states of the neurons, so the network can converge to a "normal" dynamic state, allowing for new data to be predicted [44, 45]. 


\begin{tabular}{|c|c|c|c|c|c|c|c|c|c|}
\hline & $I_{1}$ & $I_{2}$ & $I_{3}$ & $H_{1}$ & $\mathrm{H}_{2}$ & $\mathrm{H}_{3}$ & $\mathrm{H}_{4}$ & $\mathrm{H}_{5}$ & $O_{1}$ \\
\hline$I_{1}$ & $Q$ & 0 & 0 & 1 & 0 & 0 & 0 & 1 & 0 \\
\hline$I_{2}$ & 0 & $Q$ & 0 & 0 & 0 & 1 & 0 & 0 & 1 \\
\hline$I_{3}$ & 0 & 0 & & 0 & 0 & 0 & 1 & 0 & 0 \\
\hline$H_{1}$ & 0 & 0 & & 1 & 0 & 0 & 1 & 0 & 0 \\
\hline $\mathrm{H}_{2}$ & 0 & 0 & 0 & 0 & $Q$ & 1 & 0 & 0 & 0 \\
\hline $\mathrm{H}_{3}$ & 1 & 0 & 0 & 0 & 1 & $Q$ & 0 & 0 & 0 \\
\hline $\mathrm{H}_{4}$ & 0 & 1 & 0 & 0 & 0 & 0 & . & 0 & 1 \\
\hline$H_{5}$ & 0 & 0 & 0 & 1 & 0 & 0 & 0 & & 0 \\
\hline$O_{1}$ & 0 & 1 & 1 & 0 & 0 & 0 & 0 & 0 & \\
\hline
\end{tabular}

Figure 2: Recurrent neural network matrix.

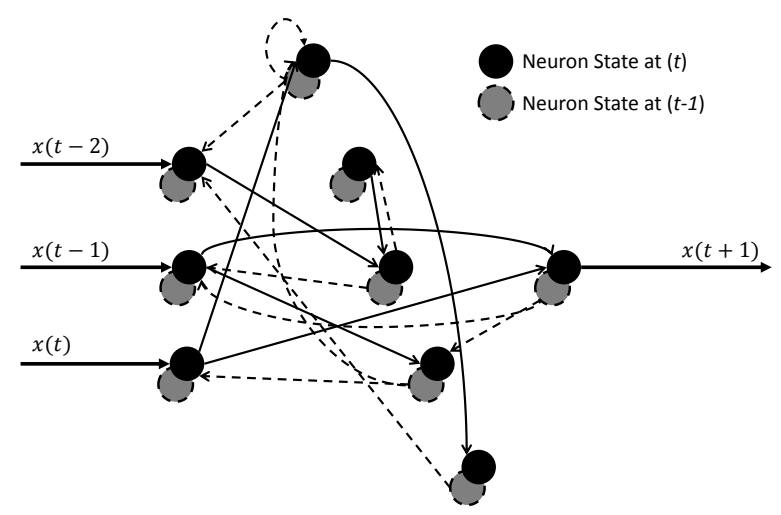

Figure 3: An example of a recurrent neural network.

A GD local search is used to fine tune the weight values of the network once it has been decoded, affecting the second chromosome of the individual. The error used during the local search is the MSE calculated on all data pairs, minus a warm-up-length and is back propagated through the network to determine the change in the weights. So any values created by the local search are compatible with the genetic algorithms crossover and mutation operators and are within an acceptable range, the local search has a bounds check on all new design variables. The new weight values are assessed after each training epoch of the local search and if a weight value is out of bounds, the weight values for all connections from the previous training epoch are used and the 
local search is stopped.

Algorithm 1 provides details of how the final Pareto set of solutions are generated.

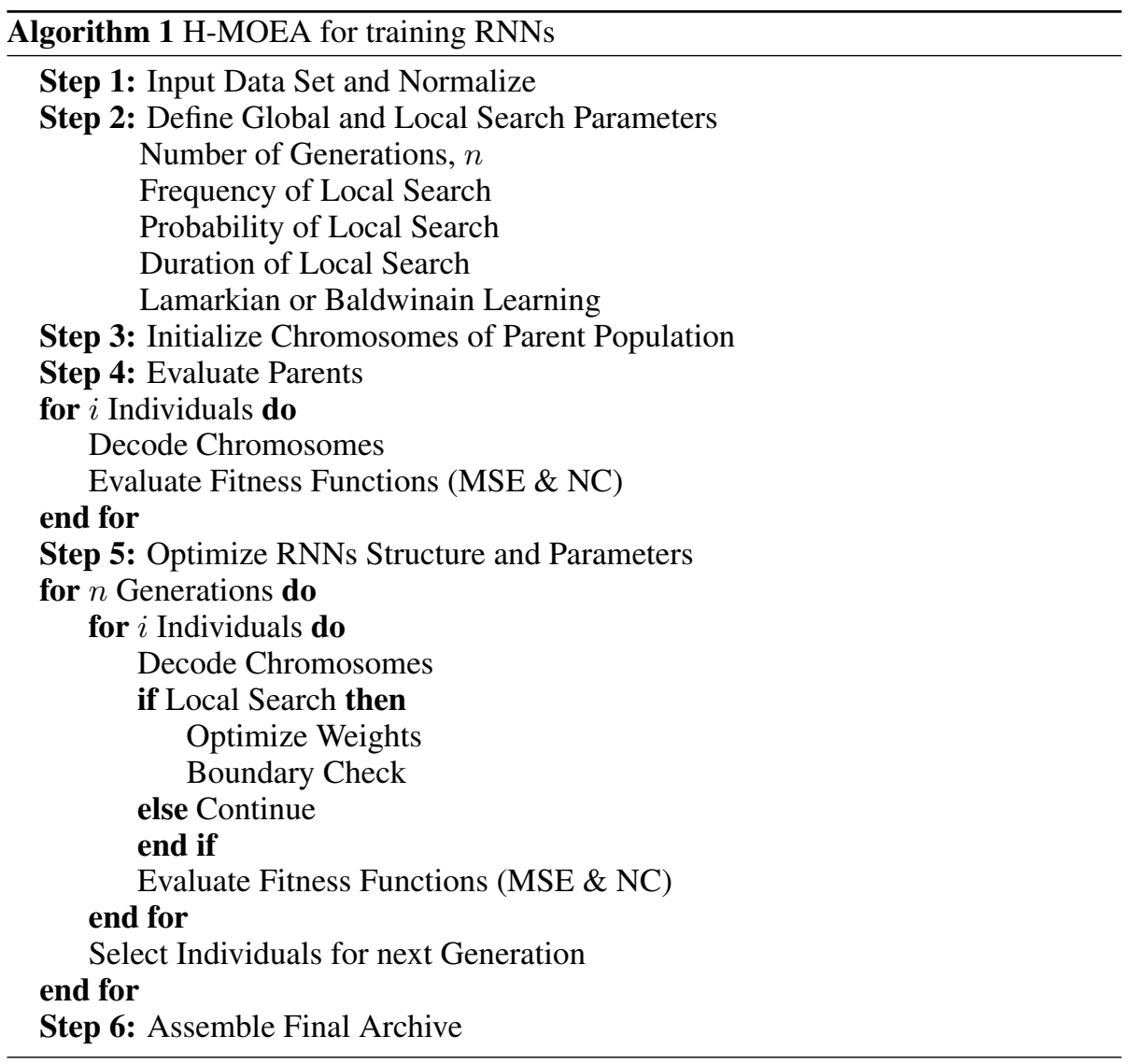

\subsection{RNN Selection and Combination}

Once the search has been completed and a Pareto set of solutions has been established, a subset of individuals in the Pareto set are selected and combined. Fig. 4 illustrates an example of a Pareto set of solutions, where each individual represents a unique RNN model. It also illustrates the selection of some solutions that can then be used in the ensemble.

Individuals at the extremes of the Pareto front are likely to represent infeasible or unsuitable networks, with one extreme representing a low complexity network and the other a large complexity network. Networks of overly low complexity exhibit a 


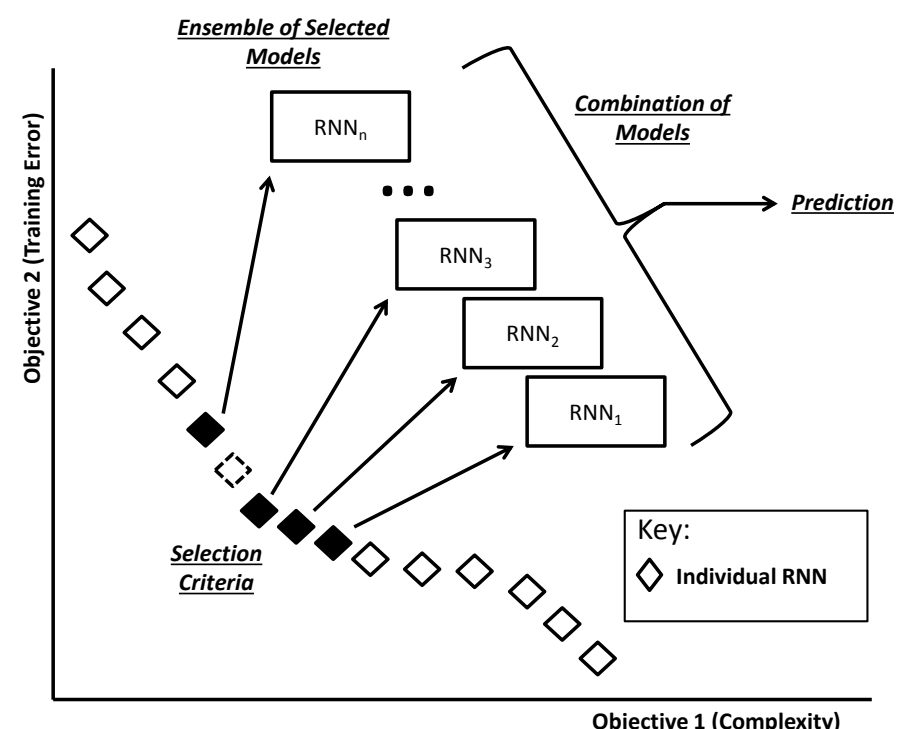

Figure 4: Pareto set of solutions.

Therefore, prior to selection it is important to filter the solutions. Only solutions below the knee point of the Pareto front will be considered for selection and the NPG is used to identify this point in the set.

The knee point of a Pareto front has been described as a "point of maximum convex bulge on the Pareto curve for a bi-criteria optimization problem" [46] or the region that "involve steep trade-off between objectives" and "high marginal rates of return" [47]. The knee point is not located at the extremes and is of interest because it is believed that the complexity of the models in this region of the Pareto front matches that of the data [14, 48] and that the models will not exhibit over-fitting on a validation data set. It is also hypothesized that models in this area will exhibit a smaller prediction variance.

The NPG was introduced in [14] to give an indication of the knee point of the Pareto front. When there is a large change in the NPG value and it gradually drops to zero, it can be said that the model complexity matches that of the data and that this is the knee-point of the Pareto front. Equation (2) is used for calculating the NPG, where 

of connections of the $i$ th and $i+1$ th Pareto-optimal solutions.

$$
N P G=\frac{M S E_{i+1}-M S E_{i}}{N C_{i}-N C_{i+1}}
$$

When the solutions are ranked in the order of increasing complexity, the following relationship holds:

$$
\begin{array}{r}
N C_{i+1}>N C_{i}, \\
M S E_{i+1} \leq M S E_{i}
\end{array}
$$

Once the knee point has been identified, four selection schemes are used.

\subsubsection{Selection Methods 1 - 3:}

In the first method, all models remaining in the Pareto-set after the initial filtering are used to create an ensemble of predictors. The second method selects members below the knee point based on their training error and the third selects those that are closest to the knee point.

\subsubsection{Selection Method 4:}

The ambiguity term of the error-ambiguity decomposition presented by Krogh and Vedelsby [15] is used to identify diverse predictors and is used as the fourth selection criterion. The ambiguity term is a measure of how an individual predictor performs compared to the ensemble of predictors, measuring the correlation or disagreement between individual networks. If the errors produced by each network are very similar, then they are strongly correlated and the ambiguity values for each network will be small. Conversely, if the networks produce different errors, then the ambiguity values will be larger. Therefore the ambiguity term in the error-ambiguity decomposition can be used as an indication of the ensemble members diversity.

Given an instance $x$, the ambiguity of an individual learner can be defined by Equation (4), with $V^{\alpha}(x)$ the output of an individual predictor, $\alpha$, on instance $x$ and $\bar{V}(x)$ 
the mean prediction output when considering all predictors, for instance $x$ :

$$
a^{\alpha}(x)=\left(V^{\alpha}(x)-\bar{V}(x)\right)^{2}
$$

This equation can be extended by taking the average over all instances as shown by Equation (5), where $p(x)$ is the distribution from which the instances are sampled:

$$
a^{\alpha}=\int a^{\alpha}(x) p(x) \mathrm{d} x
$$

\section{Experimental Results and Discussions}

This Section presents the experimental study of the H-MOEA and selection algorithms. The results are compared to other results presented in the literature for similar setups of the Mackey-Glass and Smoothed Sunspot data sets. The details of these two 


\subsection{Data sets}

\subsubsection{Mackey-Glass time series}

The Mackey-Glass time series [51] is a benchmark problem that has been widely used in the literature due to its chaotic behavior. The differential equation used to generate the time series is given in Equation (6):

$$
\frac{d x(t)}{d t}=\frac{a x\left(t-\tau_{M G}\right)}{1+x^{c}\left(t-\tau_{M G}\right)}-b x(t)
$$

The time delay parameter in Equation (6) determines the chaotic behavior of the time series, with values of $\tau_{M G}>16.8$ producing chaos. In the experiments, the following parameters were chosen so comparisons to other experiments can be made $a=0.2, b=0.1, c=10$ and $\tau_{M G}=17$. To obtain time series values at integer points, the fourth-order Runge-Kutta method was used to generate data points with a time step of 0.1 and then sampled at integer values. Initial condition $x(0)=1.2$ and $x(t)=0$ for $t<0$, for a time period of $0 \leq t \leq 1200$ is used and data points from the 118th to 1117th were extracted. The first 500 data points are used for training and the remaining 500 are used for validation. The time-series is scaled in the range $[0,1]$.

\subsubsection{Sunspot time series}

Sunspot time series data represents the solar activity of the sun. The smoothed sunspot data from the World Data Center for sunspot index [3] has been used so that suitable comparisons to the literature can be made. 2000 data points from November 1834 to June 2001 have been selected, with the first 1000 data points used for training and the second 1000 data points used for validation. The time series is scaled in the range $[-1,1]$.

\subsubsection{Performance measures}

Throughout the literature there are many different measures that have been used to evaluate the performance of models on time series data, including the mean squared error $(M S E)$, root mean squared error $(R M S E)$ and normalized mean squared error 
(NMSE). They are given in the following equations:

$$
\begin{gathered}
M S E=\frac{1}{N} \sum_{i=1}^{N}\left(y_{i}-\hat{y}_{i}\right)^{2} \\
R M S E=\sqrt{\frac{1}{N} \sum_{i=1}^{N}\left(y_{i}-\hat{y}_{i}\right)^{2}} \\
N M S E=\left(\frac{\sum_{i=1}^{N}\left(y_{i}-\hat{y}_{i}\right)^{2}}{\sum_{i=1}^{N}\left(y_{i}-\bar{y}_{i}\right)^{2}}\right)
\end{gathered}
$$

where $y_{i}, \hat{y}_{i}$ and $\bar{y}_{i}$ are the observed data, predicted data and average of observed data, respectively. $\mathrm{N}$ is the total number of data points. $\hat{y}_{i}$ is the predicted data and is the output of the ensemble. A simple averge of all ensemble members predictions is

be used as an input for the next time step. Each neuron uses the $\tanh ()$ activation function. 


\subsection{Results and Discussion}

This section reports the performance of the H-MOEA and four selection methods. sity criterion below the knee point (D) are presented. The values in brackets indicate the number of ensemble members and the highlighted values are the best performing selection method.

Table 1 Mackey-Glass Results - 500 Training.

All $=$ all models below the knee point, $\mathrm{TE}=$ selected models below the knee point based on minimum training error, $\mathrm{KP}=$ selected models below the knee point based on proximity to the knee point, $\mathrm{D}=$ selected models below the knee point based on the diversity criterion, ()$=$ ensemble size

\begin{tabular}{lcccccc}
\hline \hline \multirow{2}{*}{ Method } & \multicolumn{2}{c}{ MSE } & \multicolumn{2}{c}{ RMSE } & \multicolumn{2}{c}{ NMSE } \\
\cline { 2 - 7 } & Mean & Std Dev. & Mean & Std Dev. & Mean & Std Dev. \\
\hline All & $7.03 \mathrm{E}-05$ & $4.91 \mathrm{E}-05$ & $8.06 \mathrm{E}-03$ & $2.29 \mathrm{E}-03$ & $1.36 \mathrm{E}-03$ & $9.48 \mathrm{E}-04$ \\
TE (5) & $2.16 \mathrm{E}-04$ & $5.37 \mathrm{E}-04$ & $9.76 \mathrm{E}-03$ & $1.10 \mathrm{E}-02$ & $4.17 \mathrm{E}-03$ & $1.04 \mathrm{E}-02$ \\
TE (3) & $5.32 \mathrm{E}-04$ & $1.49 \mathrm{E}-03$ & $1.23 \mathrm{E}-02$ & $1.95 \mathrm{E}-02$ & $1.03 \mathrm{E}-02$ & $2.88 \mathrm{E}-02$ \\
KP (5) & $9.28 \mathrm{E}-05$ & $2.74 \mathrm{E}-05$ & $9.52 \mathrm{E}-03$ & $1.50 \mathrm{E}-03$ & $1.79 \mathrm{E}-03$ & $5.28 \mathrm{E}-04$ \\
KP (3) & $1.08 \mathrm{E}-04$ & $3.34 \mathrm{E}-05$ & $1.03 \mathrm{E}-02$ & $1.62 \mathrm{E}-03$ & $2.09 \mathrm{E}-03$ & $6.44 \mathrm{E}-04$ \\
D (5) & $\mathbf{5 . 7 4 E - 0 5}$ & $\mathbf{1 . 2 4 E - 0 5}$ & $\mathbf{7 . 5 3 E - 0 3}$ & $\mathbf{8 . 2 9 E - 0 4}$ & $\mathbf{1 . 1 1 E - 0 3}$ & $\mathbf{2 . 3 9 E - 0 4}$ \\
D (3) & $6.36 \mathrm{E}-05$ & $1.55 \mathrm{E}-05$ & $7.91 \mathrm{E}-03$ & $9.75 \mathrm{E}-04$ & $1.23 \mathrm{E}-03$ & $2.99 \mathrm{E}-04$ \\
\hline
\end{tabular}

It can be seen from Table 1 that an ensemble of five selected members based on the diversity criterion has performed the best for prediction mean error and standard deviation.

Fig. 5 is an example of the achieved Pareto-optinal solutions (each representing 
a RNN) and their corresponding validation performance. Selection of individuals is made from the Pareto-optimal solutions and the prediction performance of an ensemble of five members selected based on their diversity on the training data set is presented in Fig. 6

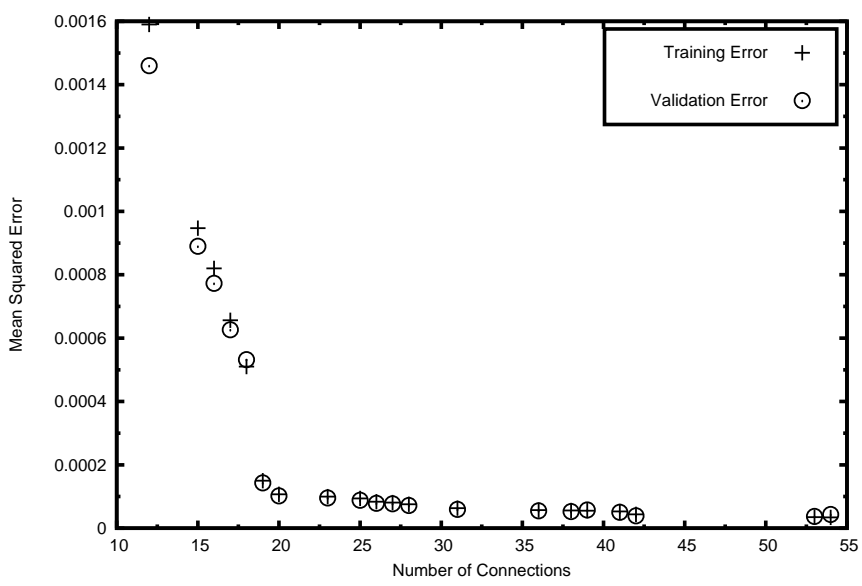

Figure 5: Mackey-Glass Pareto front - 500 Training Data Points

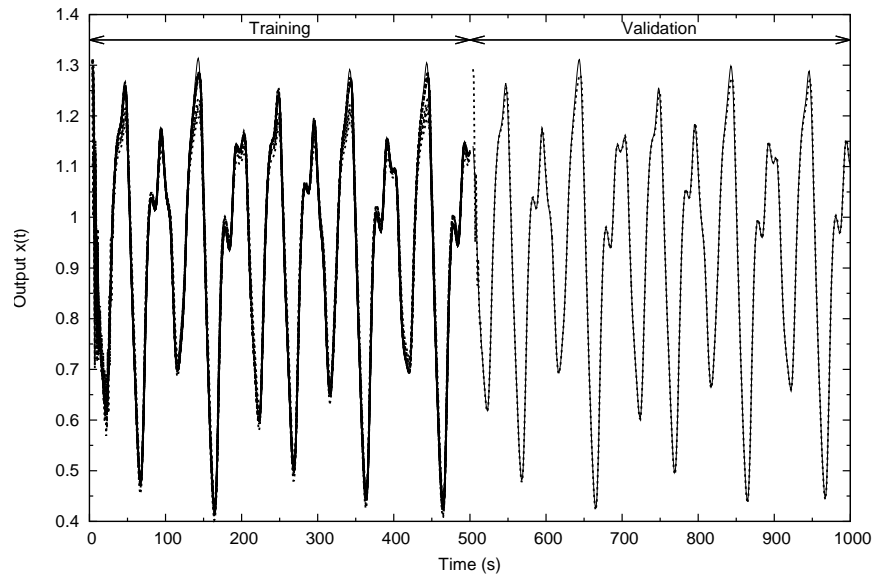

Figure 6: Mackey-Glass Prediction (The solid line is the target and the dashed lines are the prediction for training and validation data sets)

Table 2 presents the results on the Sunspot data set for the four selection methods. An ensemble of five selected members based on their proximity to the knee point has 
performed best.

Table 2 Sunspot Results - 1000 Training.

All $=$ all models below the knee point, $\mathrm{TE}=$ selected models below knee point based on minimum training error, $\mathrm{KP}=$ selected models below the knee point based on proximity to the knee point, $\mathrm{D}=$ selected models below the knee point based on the diversity criterion, ()$=$ ensemble size

\begin{tabular}{lcccccc}
\hline \hline \multirow{2}{*}{ Method } & \multicolumn{2}{c}{ MSE } & \multicolumn{2}{c}{ RMSE } & \multicolumn{2}{c}{ NMSE } \\
\cline { 2 - 7 } & Mean & Std Dev. & Mean & Std Dev. & Mean & Std Dev. \\
\hline All & $2.40 \mathrm{E}-04$ & $1.19 \mathrm{E}-04$ & $1.52 \mathrm{E}-02$ & $3.20 \mathrm{E}-03$ & $9.81 \mathrm{E}-04$ & $4.87 \mathrm{E}-04$ \\
TE (5) & $3.34 \mathrm{E}-04$ & $3.54 \mathrm{E}-04$ & $1.68 \mathrm{E}-02$ & $7.27 \mathrm{E}-03$ & $1.37 \mathrm{E}-03$ & $1.45 \mathrm{E}-03$ \\
TE (3) & $3.10 \mathrm{E}-04$ & $2.57 \mathrm{E}-04$ & $1.66 \mathrm{E}-02$ & $5.85 \mathrm{E}-03$ & $1.27 \mathrm{E}-03$ & $1.05 \mathrm{E}-03$ \\
KP (5) & $\mathbf{2 . 3 2 E - 0 4}$ & $\mathbf{2 . 6 0 E - 0 5}$ & $1.52 \mathrm{E}-02$ & $\mathbf{8 . 3 2 E - 0 4}$ & $\mathbf{9 . 4 6 E - 0 4}$ & $\mathbf{1 . 0 6 E - 0 4}$ \\
KP (3) & $2.73 \mathrm{E}-04$ & $6.87 \mathrm{E}-05$ & $1.64 \mathrm{E}-02$ & $1.91 \mathrm{E}-03$ & $1.12 \mathrm{E}-03$ & $2.81 \mathrm{E}-04$ \\
D (5) & $2.32 \mathrm{E}-04$ & $4.88 \mathrm{E}-05$ & $\mathbf{1 . 5 2 E - 0 2}$ & $1.50 \mathrm{E}-03$ & $9.47 \mathrm{E}-04$ & $1.99 \mathrm{E}-04$ \\
D (3) & $2.71 \mathrm{E}-04$ & $7.21 \mathrm{E}-05$ & $1.63 \mathrm{E}-02$ & $2.03 \mathrm{E}-03$ & $1.11 \mathrm{E}-03$ & $2.95 \mathrm{E}-04$ \\
\hline
\end{tabular}

Fig. 7 is an example of the Pareto-optimal individuals and their corresponding validation performance. The prediction performance of an ensemble of five members selected based on their proximity to the knee point is presented in Fig. 8 It can be seen in Fig. 8 that one of the models did not perform well on the training data but was kept in the Pareto set as it corresponds to the smallest network. Due to the pre-selection, this model is removed before the ensembles are created.

Tables 3 and 4 compare the best performances from Tables 1 and 2 with those reported in the literature. Only results from the literature that have used the same data sets and experimental parameters have been used in the comparison. Results are not available for all performance measures, but it is possible to compare some of the measures and it can be seen that the H-MOEA and selection methods presented are competitive with the state-of-the-art in the literature.

To further evaluate the H-MOEA and selection methods, 250 data points were used for training. Tables 5 and 6 present the results from these experiments, where it can 


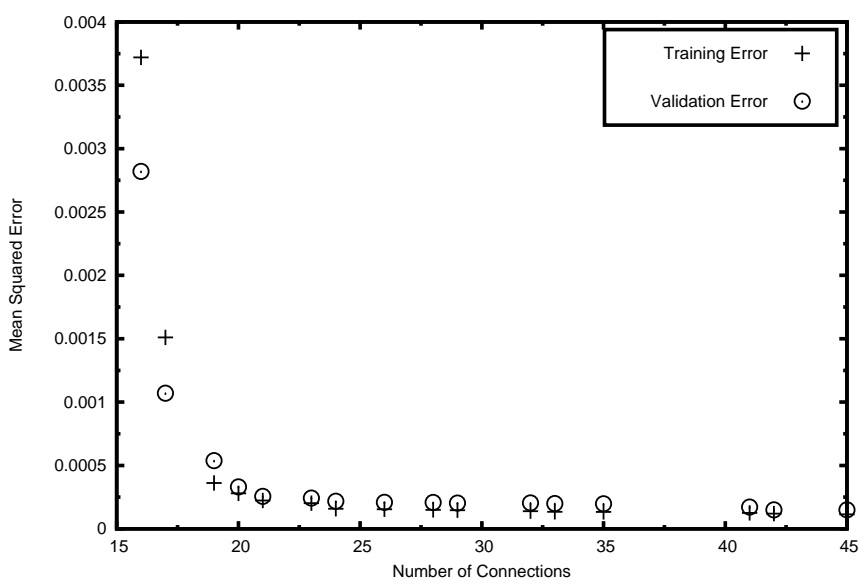

Figure 7: Sunspot Pareto front - 1000 Training Data Points

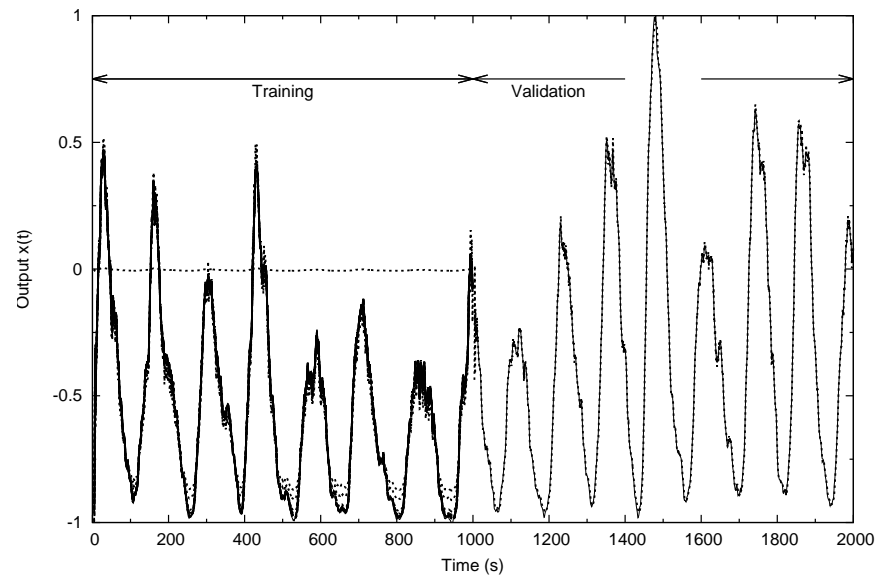

Figure 8: Sunspot Prediction (The solid line is the target and the dashed lines are the prediction for training and validation data sets)

be seen that taking a subset based on the diversity criterion has performed best for the Mackey-Glass data set and selection of individuals near the knee point of the Pareto front is best for the Sunspot data set. Comparing these results to the previous tests using more training data, these results are not as good, which is to be expected, but the same selection methods provided the best results when using less training data.

Figs. 9 and 11 present examples of the Pareto-optimal solutions when 250 training 


\begin{tabular}{|c|c|c|c|c|c|c|}
\hline \multirow[t]{2}{*}{ Model } & \multicolumn{2}{|c|}{ MSE } & \multicolumn{2}{|c|}{ RMSE } & \multicolumn{2}{|c|}{ NMSE } \\
\hline & Min & Mean & Min & Mean & Min & Mean \\
\hline RNN (CC) [17 & 4.01E-05 & - & $6.33 \mathrm{E}-03$ & $9.39 \mathrm{E}-03$ & $2.80 \mathrm{E}-04$ & $6.31 \mathrm{E}-03$ \\
\hline RNN (PSO) 18 & - & - & - & $6.25 \mathrm{E}-04$ & - & - \\
\hline RNN (GD) [19] & $1.39 \mathrm{E}-09$ & - & $3.72 \mathrm{E}-05$ & - & 2.70E-08 & - \\
\hline RNN (GA) 27] & - & 2.19E-03 & - & - & - & - \\
\hline FFNN (GA) [54] & - & - & - & - & $2.10 \mathrm{E}-02$ & - \\
\hline RNN (H-MOEA) & \multicolumn{2}{|c|}{$5.74 \mathrm{E}-05$} & \multicolumn{2}{|c|}{$7.53 \mathrm{E}-03$} & \multicolumn{2}{|c|}{$1.11 \mathrm{E}-03$} \\
\hline
\end{tabular}

\begin{tabular}{|c|c|c|c|c|c|c|}
\hline \multirow[t]{2}{*}{ Model } & \multicolumn{2}{|c|}{ MSE } & \multicolumn{2}{|c|}{ RMSE } & \multicolumn{2}{|c|}{ NMSE } \\
\hline & Min & Mean & Min & Mean & Min & Mean \\
\hline RNN (CC) 17 & $2.76 \mathrm{E}-04$ & - & $1.66 \mathrm{E}-02$ & $6.88 \mathrm{E}-02$ & $1.47 \mathrm{E}-03$ & $5.48 \mathrm{E}-02$ \\
\hline RNN (GD) [19] & $1.41 \mathrm{E}-04$ & - & $1.19 \mathrm{E}-02$ & - & $5.90 \mathrm{E}-04$ & - \\
\hline ERNN (GA) [20] & $1.66 \mathrm{E}-04$ & - & $1.29 \mathrm{E}-02$ & - & $2.80 \mathrm{E}-03$ & - \\
\hline RNN (H-MOEA) & \multicolumn{2}{|c|}{ 2.32E-04 } & \multicolumn{2}{|c|}{$1.52 \mathrm{E}-02$} & \multicolumn{2}{|c|}{$9.46 \mathrm{E}-04$} \\
\hline
\end{tabular}

Table 5 Mackey-Glass Results - 250 Training.

All $=$ all models below the knee point, $\mathrm{TE}=$ selected models below the knee point based on minimum training error, $\mathrm{KP}=$ selected models below the knee point based on proximity to the knee point, $\mathrm{D}=$ selected models below the knee point based on the diversity criterion, ()$=$ ensemble size

\begin{tabular}{lcccccc}
\hline \hline \multirow{2}{*}{ Method } & \multicolumn{2}{c}{ MSE } & \multicolumn{2}{c}{ RMSE } & \multicolumn{2}{c}{ NMSE } \\
\cline { 2 - 7 } & Mean & Std Dev. & Mean & Std Dev. & Mean & Std Dev. \\
\hline All & $3.33 \mathrm{E}-04$ & $5.55 \mathrm{E}-04$ & $1.51 \mathrm{E}-02$ & $1.03 \mathrm{E}-02$ & $6.45 \mathrm{E}-03$ & $1.07 \mathrm{E}-02$ \\
TE (5) & $6.19 \mathrm{E}-04$ & $1.01 \mathrm{E}-03$ & $2.04 \mathrm{E}-02$ & $1.43 \mathrm{E}-02$ & $1.20 \mathrm{E}-02$ & $1.96 \mathrm{E}-02$ \\
TE (3) & $8.14 \mathrm{E}-04$ & $1.54 \mathrm{E}-03$ & $2.19 \mathrm{E}-02$ & $1.83 \mathrm{E}-02$ & $1.57 \mathrm{E}-02$ & $2.98 \mathrm{E}-02$ \\
KP (5) & $3.09 \mathrm{E}-04$ & $4.78 \mathrm{E}-04$ & $1.51 \mathrm{E}-02$ & $9.03 \mathrm{E}-03$ & $5.97 \mathrm{E}-03$ & $9.25 \mathrm{E}-03$ \\
KP (3) & $2.03 \mathrm{E}-04$ & $\mathbf{9 . 0 9 E}-05$ & $1.39 \mathrm{E}-02$ & $\mathbf{3 . 0 8 E - 0 3}$ & $3.94 \mathrm{E}-03$ & $\mathbf{1 . 7 6 E}-03$ \\
D (5) & $\mathbf{1 . 7 2 E - 0 4}$ & $1.41 \mathrm{E}-04$ & $\mathbf{1 . 2 3 E - 0 2}$ & $4.49 \mathrm{E}-03$ & $\mathbf{3 . 3 3 E}-03$ & $2.74 \mathrm{E}-03$ \\
D (3) & $1.94 \mathrm{E}-04$ & $1.04 \mathrm{E}-04$ & $1.34 \mathrm{E}-02$ & $3.66 \mathrm{E}-03$ & $3.76 \mathrm{E}-03$ & $2.01 \mathrm{E}-03$ \\
\hline
\end{tabular}


Table 6 Sunspot Results - 250 Training.

All $=$ all models below the knee point, $\mathrm{TE}=$ selected models below the knee point based on minimum training error, $\mathrm{KP}$ = selected models below knee-point based on proximity to knee-point, $\mathrm{D}=$ selected models below knee-point based on diversity criteria, ()$=$ ensemble size

\begin{tabular}{lcccccc}
\hline \hline \multirow{2}{*}{ Method } & \multicolumn{2}{c}{ MSE } & \multicolumn{2}{c}{ RMSE } & \multicolumn{2}{c}{ NMSE } \\
\cline { 2 - 7 } & Mean & Std Dev. & Mean & Std Dev. & Mean & Std Dev. \\
\hline All & $3.56 \mathrm{E}-04$ & $3.09 \mathrm{E}-04$ & $1.78 \mathrm{E}-02$ & $6.10 \mathrm{E}-03$ & $1.80 \mathrm{E}-03$ & $1.57 \mathrm{E}-03$ \\
TE (5) & $1.29 \mathrm{E}-03$ & $2.56 \mathrm{E}-03$ & $2.73 \mathrm{E}-02$ & $2.34 \mathrm{E}-02$ & $6.56 \mathrm{E}-03$ & $1.30 \mathrm{E}-02$ \\
TE (3) & $2.71 \mathrm{E}-03$ & $5.71 \mathrm{E}-03$ & $3.66 \mathrm{E}-02$ & $3.71 \mathrm{E}-02$ & $1.38 \mathrm{E}-02$ & $2.90 \mathrm{E}-02$ \\
KP (5) & $\mathbf{2 . 4 5 E - 0 4}$ & $\mathbf{3 . 5 2 E - 0 5}$ & $\mathbf{1 . 5 6 E - 0 2}$ & $\mathbf{1 . 1 1 E}-03$ & $\mathbf{1 . 2 4 E - 0 3}$ & $\mathbf{1 . 7 8 E - 0 4}$ \\
KP (3) & $2.88 \mathrm{E}-04$ & $8.72 \mathrm{E}-05$ & $1.68 \mathrm{E}-02$ & $2.28 \mathrm{E}-03$ & $1.46 \mathrm{E}-03$ & $4.42 \mathrm{E}-04$ \\
D (5) & $4.28 \mathrm{E}-04$ & $5.01 \mathrm{E}-04$ & $1.89 \mathrm{E}-02$ & $8.49 \mathrm{E}-03$ & $2.17 \mathrm{E}-03$ & $2.54 \mathrm{E}-03$ \\
D (3) & $3.36 \mathrm{E}-04$ & $1.49 \mathrm{E}-04$ & $1.80 \mathrm{E}-02$ & $3.54 \mathrm{E}-03$ & $1.71 \mathrm{E}-03$ & $7.54 \mathrm{E}-04$ \\
\hline
\end{tabular}

data points are used. What can be seen in these example is that now there is less training data available, some over-fitting can be seen on a validation data set when the networks have larger complexity. This result is not unexpected and highlights why it is best not to select individual models based on the training error. Figs. 10 and 12 present examples of the prediction performance when 250 data points are used for training.

It can be seen from all the results presented that to use a subset of the individuals, after an initial selection of individuals below the knee point, is better than using all of the members below the knee point. Using the training error is not a good indicator of a suitable model and should not be used as the selection criterion. This could be due to over-fitting on larger networks. Selection based on the proximity to the knee point or the diversity criterion introduced should be used as they have shown the most successful results. Selecting five ensemble members is generally better than using three and it is clear that the standard deviation between ten runs is less when members are selected at the knee point. This is clearly shown by the sunspot data.

The algorithms presented are competitive with others in the literature. [19] is better 


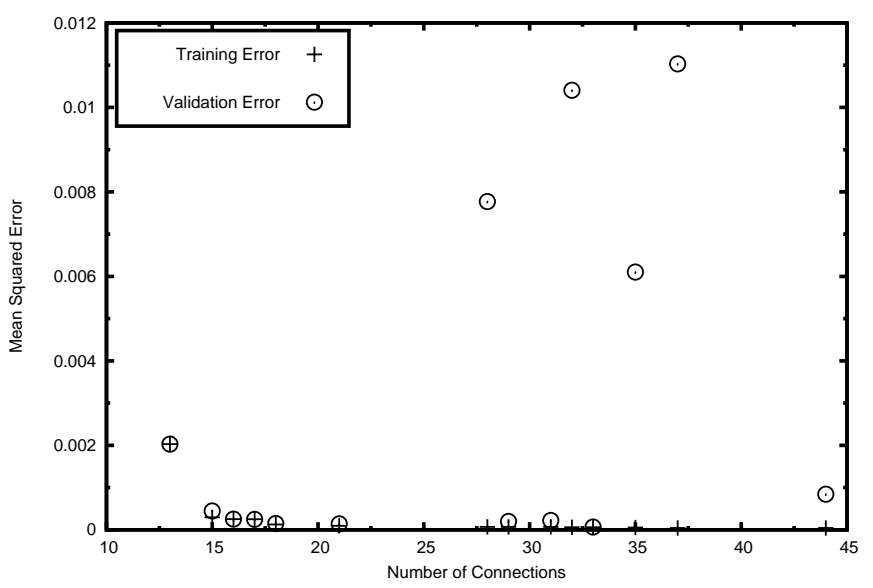

Figure 9: Mackey-Glass Pareto front - 250 Training Data Points

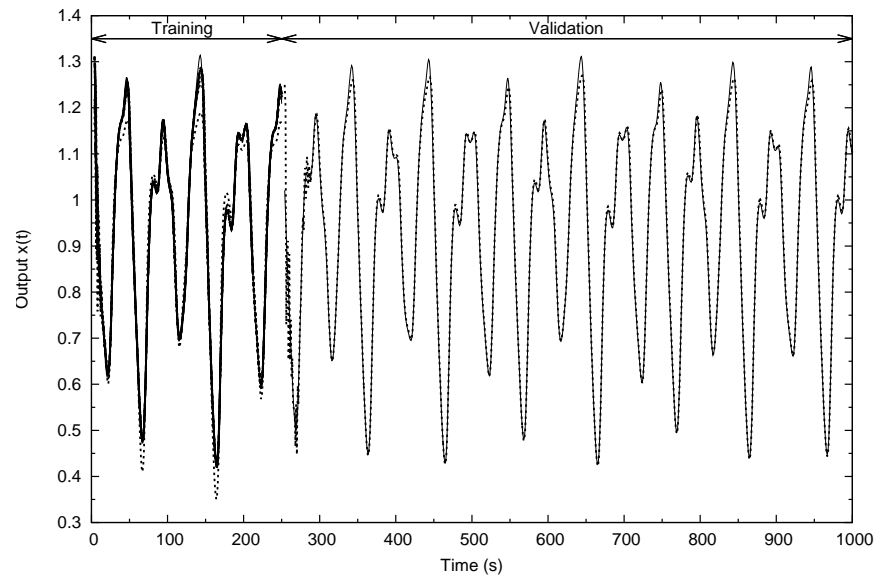

Figure 10: Mackey-Glass Prediction (The solid line is the target and the dashed lines are the prediction for training and validation data sets)

data set. It has been hard to make direct comparisons to the literature as the parameter values used in other work has not been similar and the performance measures used have been different. The best individual models have not been presented in this work and compared to others in the literature. This is because there is more confidence in a prediction made by an ensemble and this is the focus of this paper. Even when 


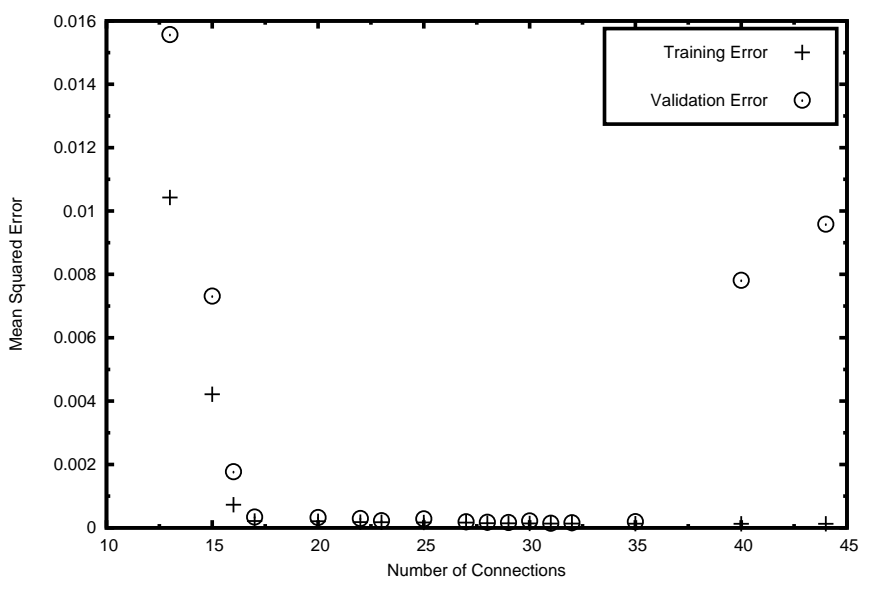

Figure 11: Sunspot Pareto front - 250 Training Data Points

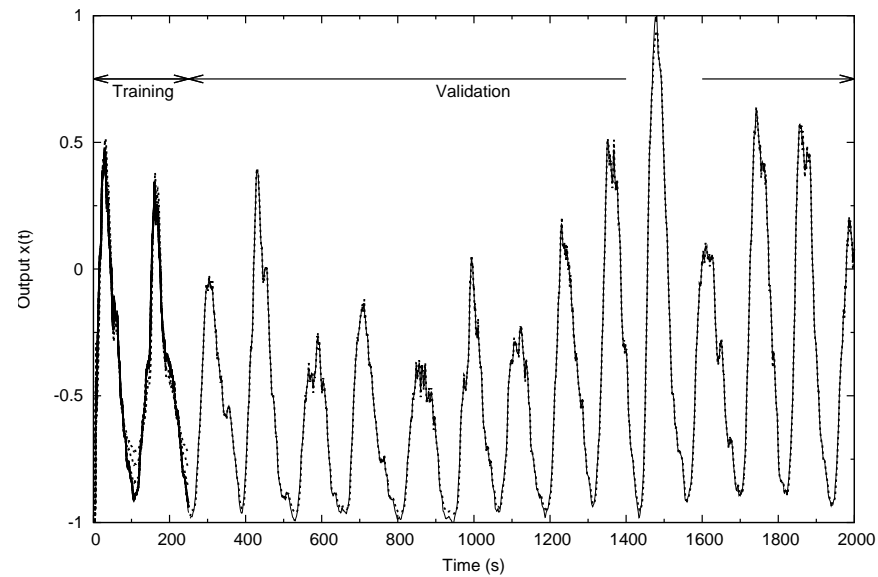

Figure 12: Sunspot Prediction (The solid line is the target and the dashed lines are the prediction for training and validation data sets)

using less training data for the sunspot data set a similar prediction performance after selection can be achieved compared to the literature, even when more data points are considered for the validation error. 


\section{Conclusions and Future Work}

that is used for training recurrent neural networks and optimizing their structure for time series prediction. The NSGA-II is used as the global optimizer to find suitable network structures and initial starting weights for the networks and the IRProp+ gradient descent algorithm is used as a local search to fine tune the weights. The result is a set of Pareto-optimal individual networks that can be used to construct an ensemble of predictors. Four selection schemes have been investigated after an initial filtering of the fronts to only include individuals below the knee point of the Pareto front. The normalized performance gain was used to identify the knee point of the Pareto front.

The Mackey-Glass and smoothed Sunspot data sets have been used to evaluate the H-MOEA and selection criteria. Two different setups for each data set have been investigated with different sizes of training and validation data sets.

It can be concluded from this work that the developed H-MOEA is competitive with the other models in the literature. Also, selecting a subset of individuals below the knee point is better than sampling all members below the knee point, with selection of models that are located near the knee point and models that are diverse on the training data performing better than models selected based on their specific training error. Ensembles with five members are better than those with three members. It should be noted though, that the performance between all selection methods is not substantially different.

A simple average has been been used to combine the individual predictors at each

This paper has introduced a hybrid multi-objective evolutionary algorithm (H-MOEA) data point, but different combination methods could be investigated in the future. The algorithms presented will also be applied to large-scale time series prediction, by predicting a number of steps ahead, instead of the one step ahead that has been used in this work. This is related to the reconstruction of the data, which means different values of $\tau$ and $D$ should be investigated.

Another future work is the application of the developed H-MOEA and ensemble member selection methods to complex engineering problems such as the prediction of computational fluid dynamic convergence data, which is a long-term prediction task 
that can be used to reduce computational time in evolutionary optimization of expensive problems [55].

\section{References}

[1] I. A. Gheyas, L. S. Smith, A novel neural network ensemble architecture for time series forecasting, Neurocomputing 74 (18) (2011) 3855-3864.

[2] H. S. Hippert, C. E. Pedreira, R. C. Souza, Neural networks for short-term load forecasting: A review and evaluation, IEEE Transactions on Power Systems 16 (1) (2001) 44-55.

[3] SIDC-team, The international sunspot number, Monthly Report on the International Sunspot Number, online catalogue, http://www.sidc.be/sunspot-data/.

[4] D. Kim, C. Kim, Forecasting time series with genetic fuzzy predictor ensemble, IEEE Transactions on Fuzzy Systems 5 (4) (1997) 523-535.

[5] M. R. Hassan, B. Nath, M. Kirley, J. Kamruzzaman, A hybrid of multiobjective evolutionary algorithm and hmm-fuzzy model for time series prediction, Neurocomputing 81 (0) (2012) $1-11$.

[6] G. Huang, L. Wang, Hybrid neural network models for hydrologic time series forecasting based on genetic algorithm, in: Computational Sciences and Optimization (CSO), 2011 Fourth International Joint Conference on, 2011, pp. 13471350.

[7] X. Yao, M. M. Islam, Evolving artificial neural network ensembles, IEEE Computational Intelligence Magazine 3 (1) (2008) 31-42.

[8] L. K. Hansen, P. Salamon, Neural network ensembles, IEEE Trans. Pattern Anal. Mach. Intell. 12 (10) (1990) 993-1001.

[9] X. Yao, Y. Liu, Making use of population information in evolutionary artificial neural networks, IEEE Transactions on Systems, Man, and Cybernetics, Part B: Cybernetics 28 (3) (1998) 417-425. 
[15] A. Krogh, J. Vedelsby, Neural network ensembles, cross validation, and active learning, in: Advances in Neural Information Processing Systems, MIT Press, 1995, pp. 231-238.

[16] S. Haykin, Neural Networks and Learning Machines (3rd Edition), 3rd Edition,

[17] R. Chandra, M. Zhang, Cooperative coevolution of elman recurrent neural networks for chaotic time series prediction, Neurocomputing 86 (2012) 116 - 123.

[18] X. Wang, L. Ma, B. Wang, T. Wang, A hybrid optimization-based recurrent neural network for real-time data prediction, Neurocomputing 120 (2013) 547 - 559.

[19] M. Ardalani-Farsa, S. Zolfaghari, Chaotic time series prediction with residual analysis method using hybrid elmannarx neural networks, Neurocomputing 73 (13-15) (2010) 2540 - 2553. 
[20] Q. Ma, Q. Zheng, H. Peng, T. Zhong, L. Xu, Chaotic time series prediction based on evolving recurrent neural networks, in: International Conference on Machine Learning and Cybernetics, Vol. 6, 2007, pp. 3496-3500.

[21] Y.-C. Lin, Y.-C. Lin, K.-L. Su, Evolutionary neural networks for time series prediction, in: International Conference on Genetic and Evolutionary Computing (ICGEC), 2010, pp. 219-223.

[22] R. Zemouri, D. Racoceanu, N. Zerhouni, Recurrent radial basis function network for time-series prediction, Engineering Applications of Artificial Intelligence 16 (2003) 453-463.

[23] K. Lukoseviciute, M. Ragulskis, Evolutionary algorithms for the selection of time lags for time series forecasting by fuzzy inference systems, Neurocomputing 73 (10-12) (2010) 2077 - 2088.

[24] B. A. Pearlmutter, Gradient calculations for dynamic recurrent neural networks: A survey, IEEE Transactions on Neural Networks 6 (5) (1995) 1212-1228.

[25] M. Han, J. Xi, S. Xu, F.-L. Yin, Prediction of chaotic time series based on the recurrent predictor neural network, IEEE Transactions on Signal Processing 52 (12) (2004) 3409-3416.

[26] A. Blanco, M. A. Delgado, M. C. Pegalajar, A real-coded genetic algorithm for training recurrent neural networks, Neural Networks 14 (1) (2001) 93 - 105.

[27] A. A. Ferreira, T. B. Ludermir, R. R. de Aquino, Comparing recurrent networks for time-series forecasting, in: The 2012 International Joint Conference on Neural Networks (IJCNN), 2012, pp. 1-8.

[28] M. P. Cuellar, M. A. Delgado, M. C. Pegalajar, Topology optimization and training of recurrent neural networks with Pareto-based multi-objective algorithms: A experimental study, in: Computational and Ambient Intelligence, Vol. 4507 of Lecture Notes in Computer Science, Springer, 2007, pp. 359-366. 
[29] H. Chihi, N. Arous, Recurrent neural networks design by means of multiobjective genetic algorithm, International Journal of Computer Science 8 (1) (2011) 296-302.

[30] M. A. Delgado, M. P. Cuellar, M. C. Pegalajar, Multiobjective hybrid optimization and training of recurrent neural networks, IEEE Transactions on Systems, Man, and Cybernetics, Part B: Cybernetics 38 (2) (2008) 381-403.

[31] J. H. Ang, C. K. Goh, E. J. Teoh, A. A. Mamun, Multi-objective evolutionary recurrent neural networks for system identification, in: IEEE Congress on Evolutionary Computation, 2007, pp. 1586-1592.

[32] N. Subrahmanya, Y. C. Shin, Constructive training of recurrent neural networks using hybrid optimization, Neurocomputing 73 (13-15) (2010) 2624-2631.

[33] K. W. Ku, M.-W. Mak, W.-C. Siu, A study of the lamarckian evolution of recurrent neural networks, IEEE Transactions on Evolutionary Computation 4 (1) (2000) 31-42.

[34] X. Yao, Evolving artificial neural networks, Proceedings of the IEEE 87 (9) (1999) 1423-1447.

[35] Z.-H. Zhou, Ensemble Methods: Foundations and Algorithms, Chapman \& Hall/CRC Data Mining and Knowledge Discovery Serie, Taylor \& Francis, 2012.

[36] G. Brown, J. Wyatt, R. Harris, X. Yao, Diversity creation methods: a survey and categorisation, Journal of Information Fusion 6 (1) (2005) 1 - 28.

[37] C. A. C. Coello, G. B. Lamont, D. A. van Veldhuizen, Evolutionary Algorithms for Solving Multi-Objective Problems, 2nd Edition, Springer, 2007.

[38] Z.-H. Zhou, J. Wu, W. Tang, Ensembling neural networks: Many could be better than all, Artificial Intelligence 137 (2002) 239-263.

[39] D. Ruta, B. Gabrys, Neural network ensembles for time series prediction, in: International Joint Conference on Neural Networks, 2007, pp. 1204-1209. 
[40] D. K. Barrow, S. F. Crone, N. Kourentzes, An evaluation of neural network ensembles and model selection for time series prediction, in: International Joint Conference on Neural Networks, 2010, pp. 1-8.

[41] H. Yang, J. Shi, A three-stage SVM ensemble algorithm for chaotic time series prediction, in: 2010 Second International Workshop on Education Technology

[48] Y. Jin, R. Gruna, B. Sendhoff, Pareto analysis of evolutionary and learning systems, Frontiers of Computer Science in China 3 (1) (2009) 4-17.

[49] E. Sanchez, S. Pintos, N. V. Queipo, Toward an optimal ensemble of kernel-based approximations with engineering applications, in: International Joint Conference on Neural Networks, 2006, pp. 2152 -2158. 
525 [50] Y. Jin, B. Sendhoff, Reducing fitness evaluations using clustering techniques and neural networks ensembles, in: Proceedings of the Genetic and Evolutionary Computation Conference, Springer, 2004, pp. 688-699.

[51] M. C. Mackey, L. Glass, Oscillation and chaos in physiological control systems, Science 1977 (4300) (1977) 287-289.

[52] K. Deb, A. Pratap, S. Agarwal, T. Meyarivan, A fast and elitist multiobjective genetic algorithm: NSGA-II, IEEE Transactions on Evolutionary Computation 6 (2) (April 2002) 182-197.

[53] C. Igel, M. Husken, Empirical evaluation of the improved Rprop learning algorithms, Neurocomputing 50 (2003) $105-123$.

[54] S. Paul, S. Kumar, L. Singh, Novel hybrid compact genetic algorithm for simultaneous structure and parameter learning of neural networks, in: IEEE Congress on Evolutionary Computation (CEC), 2012, pp. 1-6.

[55] Y. Jin, Surrogate-assisted evolutionary computation: Recent advances and future challenges, Swarm and Evolutionary Computation 1 (2) (2011) 61 - 70. 